\title{
A Rare Cause of Ventricular Fibrillation Confirmed by Speckle-Tracking Imaging
}

\author{
Larisa Anghel ${ }^{1,2}$, Eduard Dabija ${ }^{1}$, Liviu Macovei ${ }^{1,2}$, Cristina Prisacariu ${ }^{1,2}$, Mihaela Viviana Ivan ${ }^{3}$, \\ Cătălina Arsenescu Georgescu ${ }^{1,2}$
}

1 "Prof. Dr. George I.M. Georgescu" Cardiovascular Diseases Institute, Iaşi, Romania

2 "Grigore T. Popa" University of Medicine and Pharmacy, Iaşi, Romania

3 "Victor Babeş" University of Medicine and Pharmacy, Timişoara, Romania

\section{ABSTRACT}

Introduction: Cardiac arrhythmias caused by electrical injuries are rare among emergency service admittances. We present a case of ventricular fibrillation (VF) with a rare etiology, confirmed by speckle-tracking imaging. Case presentation: A 38-year-old man was addressed to our hospital to evaluate the etiology of an episode of $\mathrm{VF}$, promptly resuscitated in a territorial hospital. On admission, his 12-lead electrocardiogram revealed a sinus rhythm, without any ST-T changes or atrioventricular conduction disorders. Transthoracic echocardiography and coronary angiography were normal, and the electrophysiological study did not induce VF. Anamnesis showed that the arrhythmia occurred after an electrical injury, resulting from the contact with a domestic low-voltage source. Speckle-tracking imaging revealed closure of the electric arc within the heart, which could explain the absence of skin-burn injuries in this case. The patient was discharged after seven days of hospitalization, without any complication. Conclusions: VF can occur after an unexpected electrical shock during a household accident. This case report underlines the need for a complex interdisciplinary approach in such difficult cases, when the absence of any electrical injuries on the skin makes it difficult to recognize the electrical shock that triggered the ventricular fibrillation.

Keywords: ventricular fibrillation, electrocution, skin injuries, speckle tracking

\section{ARTICLE HISTORY}

Received: November 25, 2017

Accepted: December 10, 2017

\section{Larisa Anghel}

Blv. Carol I nr. 50

700503 Iași, Romania

Tel: +40 232211834

E-mail: larisa_med86@yahoo.com

\section{INTRODUCTION}

Ventricular arrhythmias are the major cause of morbidity and mortality in patients with structural heart disease. At the same time, this severe type of rhythm disorders can be responsible for sudden cardiac death in patients with structurally normal hearts. ${ }^{1,2}$ Overall, the main causes of sudden unexpected death are represented by ventricular tachycardia and ventricular fibrillation (VF). One of the rare causes of VF in patients with a structurally normal heart is represented by electrical injuries. ${ }^{3,4}$ The management challenge in these cases is to deal not only with the VF as the presenting symptom, but also with the pervading risk of sudden cardiac death that may represent the consequence of a pre-existing arrhythmogenic substrate. 5,6 


\section{CASE REPORT}

We present the case of a 38-year-old man, addressed to our hospital to evaluate the etiology of an episode of VF, promptly resuscitated in a territorial hospital, without any neurological sequelae. There was no history of angina, dyspnea, or drug intake. The patient had a 10-year history of smoking, occasional alcohol intake, and no illicit drug use.

On physical examination, the patient was hemodynamically stable, with a blood pressure of $125 / 80 \mathrm{mmHg}$, a peripheral pulse of $75 \mathrm{bpm}$, normal saturation on room air, and without any skin injuries. He looked pale and anxious. Cardiac examination showed normal heart sounds with regular rate and rhythm, and no jugular venous distention. Arterial pulse was strong and equal in all limbs, there was no edema in the lower extremities, and the lungs were clear. Examinations of cardiac, neurologic, respiratory and gastrointestinal systems showed normal relations. The patient was subjected to standard baseline investigations and was admitted to the Intensive Cardiac Care Unit for continuous cardiac monitoring. His 12-lead electrocardiogram revealed a sinus rhythm, without any ST-T changes or atrioventricular conduction disorders (Figure 1).

Except for a mild increase of creatine kinase $\mathrm{MB}$ (CK-MB $48 \mathrm{U} / \mathrm{L}$ ) and liver enzymes (ALAT $116 \mathrm{U} / \mathrm{L}$, ASAT $123 \mathrm{U} / \mathrm{L}$ ), his blood tests, including Troponin-I, complete blood count and biochemical profile, were normal. Serum levels of $\mathrm{K}^{+}$were also normal $(4 \mathrm{mmol} / \mathrm{L})$. His chest $\mathrm{X}$-ray was normal, and echocardiography showed a mildly dilated left ventricle with normal wall thickness and function. No significant valvular abnormalities were detected.
Troponin-I was repeated at 6 and 12 hours, but all turned out to be negative. The abdominal ultrasound revealed a fatty liver, without other liver or gallbladder disease.

In order to elucidate the cause of VF in this young smoker patient, without any family or personal history of cardiovascular disease and in the absence of any electrolyte disorder, we performed a coronary angiography in emergency settings, which revealed normal epicardial coronary arteries (Figure 2).

Holter ECG monitoring performed during hospitalization in the absence of an antiarrhythmic medication did not reveal the presence of sustained ventricular arrhythmias.

An electrophysiological study with programmed ventricular stimulation was used to document the inducibility of $\mathrm{VF}$, guide ablation, assess the risks of recurrent ventricular arrhythmia or sudden cardiac death, evaluate loss of consciousness in this patient and assess the indication for implantable cardioverter defibrillator therapy. The electrophysiological study revealed a normal atrioventricular conduction, without any inducible atrial or ventricular tachyarrhythmias.

In order to elucidate the cause of $\mathrm{VF}$, we resumed the anamnesis with the patient's family, who said that during the onset of arrhythmia, he was working on an improvised electrical installation. Therefore, a household accident with electrocution was considered the etiology of the patient's arrhythmia. Interestingly, the patient had no electrical skin injuries which could have helped us to elucidate more quickly the cause of VF.

We have also evaluated the myocardial injury of this patient, which was difficult in the absence of typical chest

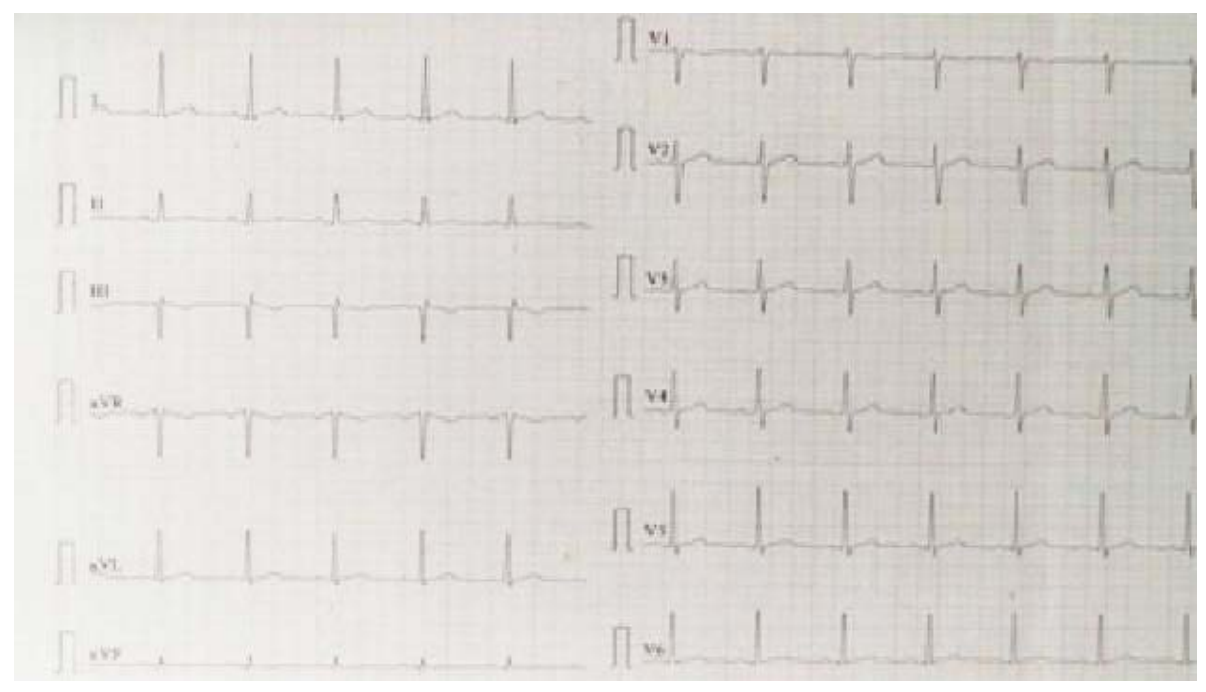

FIGURE 1. The 12-lead electrocardiogram at presentation showing sinus rhythm, without any ST-T changes or atrioventricular conduction disorders 

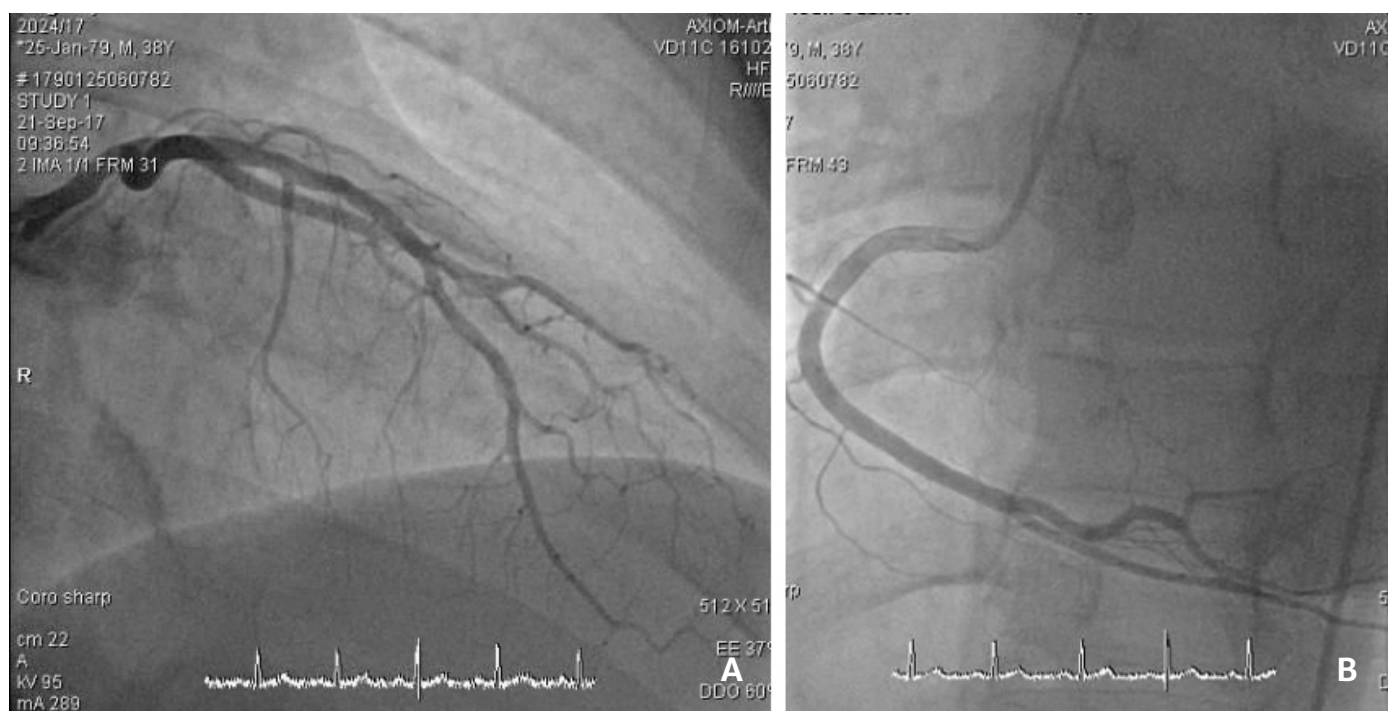

FIGURE 2. Coronary angiography showing normal left (A) and right (B) coronary arteries

pain and lack of specific changes on electrocardio-graphy. The current golden standard for assessment of left ventricular systolic function is represented by determination of ejection fraction. However, we assessed the changes in myocardial function using 2D speckle traking imaging, with both strain and strain rate measures, as basic descriptors characterizing the nature and function of myocardial tissue. Speckle-tracking imaging revealed closure of the electric arc within the heart, which could explain the absence of skin lesions in our patient (Figure 3).

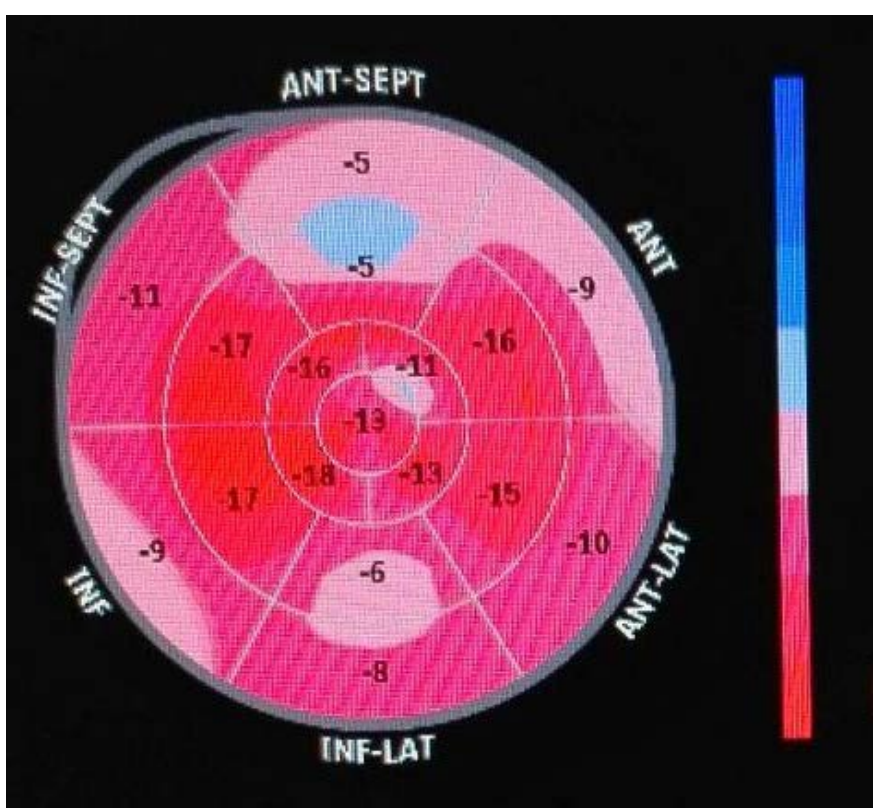

FIGURE 3. Speckle-tracking imaging revealing the closure of the electric arc within the heart
The patient was discharged without any complication after seven days of hospitalization, without any treatment at home, but with the recommendation to return after a month for reassessment and follow-up.

Publication of the data has been approved by the patient and by the institution where he was admitted.

\section{DISCUSSIONS}

The heart is one of the most susceptible organs to electrical injury. Electrical shock may cause direct myocardial necrosis or cardiac arrhythmias, and asystole and VF are the most severe arrhythmic complications of electrical injury. ${ }^{7,8}$ Increased cardiac sodium/potassium pump activities and an increase of serum potassium concentration may trigger arrhythmias after electrical injury. ${ }^{9}$

Although ventricular fibrillation is one of the common arrhythmias in patients with electrical injury, in most of the cases there are some tissue lesions which help us to diagnose and manage all the cases on time. ${ }^{10,11}$ Particular in this case was the absence of any skin injury and the impossibility of a proper anamnesis, which delayed the correct diagnosis and therapeutic management of the patient.

Even though electrocution has been recognized and studied for over a century, many secrets remain about the mechanism of occurrence of ventricular arrhythmias. The electrical injury of greatest concern is lethal ventricular arrhythmia, especially ventricular fibrillation..$^{1,12}$

There are three means of inducing VF with electrical currents in the healthy heart. The first is the delivery of "shock on T", which involves delivering a single strong 
electrical pulse during the time of the T-wave to instantly cause VF. ${ }^{9,12}$ The second method, known as "electrocution" in classical terms, is based on an electrically-induced VF mechanism. It requires capture of an extremely rapid cardiac rhythm, typically $>450$ beats per minute, which induces VF within a few seconds in a normal heart. This method takes far less current than "T-shock" induction, but also several pulses (typically at least 6 pulses). ${ }^{13,14}$ The third method of inducing VF is by long-term highrate cardiac capture causing sufficient ischemia to lower the VF threshold and to allow the induction of VF.9,14

After resuscitation, patients should be monitored for arrhythmia. Serial enzymes and echocardiography may help to estimate the extent of myocardial injury. Patients with significant myocardial damage should be monitored closely for complications. ${ }^{15}$ Cardiac arrhythmias due to electrical injuries are usually observed during or immediately after the event. Cardiac monitoring is recommended if there is loss of consciousness or documented rhythm disturbance and in case of abnormal ECG at the time of admission. ${ }^{2}$

Coronary angiography is an extremely useful diagnostic tool, playing an important role in establishing or excluding the presence of significant obstructive coronary artery disease in patients with life-threatening ventricular arrhythmias or in survivors of sudden cardiac death.

The mortality associated with domestic electrocution is difficult to estimate, due to the variable severity of the shock. However, it has been demonstrated that for every three significant injuries, there is approximately one death. $^{2}$ ECG abnormalities associated with electrocution usually resolve in a few weeks, and ventricular dysfunction also improves in most patients, who tend to have a good long-term prognosis. However, survivors of this kind of cardiac arrest should be monitored periodically for at least one year. ${ }^{15,16}$

\section{CONCLUSIONS}

This case represents a diagnostic and therapeutic challenge for practitioner physicians. VF can occur after an unexpected electrical shock during a household accident, and these patients require a proper monitoring, especially in the presence of a documented rhythm disorder, loss of consciousness, or abnormal ECG at the time of admission. The case presented underlines the need for a complex interdisciplinary approach in such difficult cases, when the absence of electrical skin injuries makes it difficult to recognize the electrical shock that triggered the ventricular fibrillation.

\section{CONFLICT OF INTEREST}

Nothing to declare.

\section{ACKNOWLEDGEMENT}

The study was not supported by granting or commercial sources of funding.

\section{REFERENCES}

1. Fish RM. Electrical injuries. In: Tintinalli JE, Kelen GD, Stapczynski JS, Ma OJ, Cline DM, eds. Emergency Medicine: A Comprehensive Study Guide. 6th ed. New York, NY: McGrawHill, 2004; p. 1231-1235.

2. Arnoldo B, Klein M, Gibran NS. Practice guidelines for the management of electrical injuries. J Burn Care Res. 2006;27:439-447. doi: 10.1097/01.BCR.0000226250.26567.4C.

3. Dresen WF, Ferquson JD. Ventricular arrhythmias. Cardiol Clin. 2018;36:129-139. doi: 10.1016/j.ccl.2017.08.007.

4. Fish RM. Electric injury: part II. Specific injuries. J Emerg Med. 2000;18:27-34.

5. Akkaş M, Hocagil H, Ay D, Erbil B, Kunt MM, Ozmen MM. Cardiac monitoring in patients with electrocution injury. Ulus Travma Acil Cerrahi Derg. 2012;18:301-305. doi: 10.5505/ tjtes.2012.69158.

6. Kondur AK, Afonso LC, Berenbom LD, Lakkireddy DR. Implantable cardioverter defibrillators save lives from lightning-related electrocution too! Pacing Clin Electrophysiol. 2008;31:256-257. doi: 10.1111/j.1540-8159.2007.00980.x.

7. Donoghue E, Lifschultz B. Electrical and Lightning Injuries. In: Spitz W, ed. Medicolegal Investigation of Death. 4 ed. Springfield, 2006; p. 882-901.

8. Sharma AD, Fain E, O'Neill PG, et al. Shock on T versus direct current voltage for induction of ventricular fibrillation: a randomized prospective comparison. Pacing Clin Electrophysiol. 2004;27:89-94.

9. Kroll MW, Panescu D, Hinz AF, Lakkireddy D. A novel mechanism for electrical currents inducing ventricular fibrillation: The three-fold way to fibrillation. Conf Proc IEEE Eng Med Biol Soc. 2010;1:1990-1996. doi: 10.1109/ IEMBS.2010.5627490.

10. Link MS, Estes NA 3rd. Mechanically induced ventricular fibrillation (commotio cordis). Heart Rhythm. 2007;4:529532. doi: 10.1016/j.hrthm.2006.12.020.

11. Mockel M, Searle J, Muller R, et al. Chief complaints in medical emergencies: do they relate to underlying disease and outcome? The Charite Emergency Medicine Study (CHARITEM). Eur J Emerg Med. 2013;20:103-108. doi: 10.1097/ MEJ.obo13e328351e609.

12. Bailey B, Forget S, Gaudreault P. Prevalence of potential risk factors in victims of electrocution. Forensic Sci Int. 2001;123:58-62.

13. Tang PT, Shenasa M, Boyle NG. Ventricular arrhythmias and sudden cardiac death. Card Electrophysiol Clin. 2017; 9:693708. doi: 10.1016/j.ccep.2017.08.004.

14. Fatovich DM. Delayed lethal arrhythmia after an electrical injury. Emerg Med J. 2007;24:743. doi: 10.1136/ emj.2007.050245. 
15. Bailey B, Gaudreault P, Thivierge RL. Cardiac monitoring of high-risk patients after an electrical injury: a prospective multicentre study. Emerg Med J. 2007;24:348-352. doi: 10.1136/emj.2006.044677.

16. Lappegard KT. Induction of ventricular tachycardia by alternate current due to an insufficiently grounded electrical system. Pacing Clin Electrophysiol. 2012;35:e170-172. doi: 10.1111/j.1540-8159.2010.02964.x. 